\title{
Überleitungsmanagement optimieren
}

_ In ländlichen Regionen sind qualifizierte Schmerztherapeuten noch immer rar, weshalb der Hausarzt meistens der erste Ansprechpartner ist, erläuterte Dr.

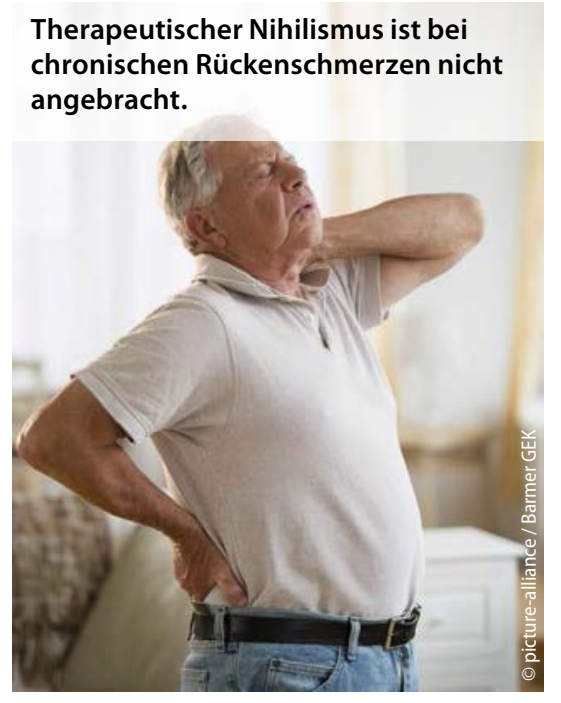

Martina Bida, Lebus. „Häufig kommen Patienten mit starken Beschwerden zu mir in der Praxis, die entweder durch Selbstmedikation oder von anderen Ärzten erfolglos mit NSAR vorbehandelt sind“, so ihre Erfahrung.

Bida setzt bei chronischem Rückenschmerz bevorzugt Opioide wie Tapentadol in Retardform (Palexia ${ }^{\star}$ retard) ein, das sich durch eine gute Schmerzlinderung auszeichnet. Die gastrointestinale Verträglichkeit sei ihrer Erfahrung nach besser als bei anderen Opioiden. Vorteilhaft für Patienten mit Polypharmazie ist, dass bei der Verstoffwechslung von Tapentadol keine aktiven Metaboliten entstehen. Als Vorteil sieht Bida auch den dualen Wirkmechanismus: Neben der Wirkung an $\mu$-Opioidrezeptoren agiert Tapentadol auch als Noradrenalin-Wiederaufnahmehemmer und stärkt so die körpereigene Schmerzhemmung.
Für Patienten, die in einer Klinik oder Schmerzambulanz erfolgreich auf Tapentadol eingestellt wurden, ist die Weiterverordnung durch den Hausarzt wünschenswert. Im Falle von Tapentadol ist die vom SGB V geforderte wirtschaftliche Nachverordnung durch zahlreiche Rabattverträge gewährleistet, erläuterte Johannes Krings, Grünenthal. Laut Dr. Thomas Hofmann, Dortmund, ist es jedoch sehr wichtig, dass die erzielten Therapieerfolge gut dokumentiert werden und dass sich im Entlassbrief eine klare rationale Begründung für den Einsatz von Tapentadol findet. Es sollte auch aufgeführt werden, dass andere Optionen nicht zum Therapieerfolg geführt haben.

\section{Dr. Claudia Bruhn}

- Fachpressegespräch „Wenn Kliniker und Niedergelassene an einem Strang ziehen: Arbeitsfähigkeit erhalten - starke Rückenschmerzen mit Begleiterkrankungen früh und effektiv behandeln", Berlin, Mai 2017 (Veranstalter: Grünenthal)

\section{Analoges Basalinsulin bei Typ-2-Diabetes}

\section{Weniger Hypoglykämien}

_ Bei Menschen mit Typ-2-Diabetes, die ein Basalinsulin benötigen, ermöglicht Toujeo (Insulin glargin $300 \mathrm{E} / \mathrm{ml}$ ) eine effektive Blutzuckerkontrolle, die im Vergleich zu Insulin glargin $100 \mathrm{E} / \mathrm{ml}$ (Lantus ${ }^{\oplus}$ ) mit einem geringeren Risiko für Unterzuckerungen - insbesondere während der Nacht - assoziiert ist. Das erklärte Prof. Petra-Maria SchummDraeger, München.

Dem stimmte Prof. Hellmut Mehnert, München, zu. Er betonte: „Insulinanaloga ahmen die physiologische, körpereigene Insulinsekretion nach.“ Sowohl Insulin glargin $300 \mathrm{E} / \mathrm{ml}$ als auch Insulin glargin $100 \mathrm{E} / \mathrm{ml}$ bräcuhten in der Regel nur einmal pro Tag gespritzt zu werden, wobei das länger wirkende Insulin glargin $300 \mathrm{E} / \mathrm{ml}$ bei Menschen mit Typ-2-Diabetes wegen der geringeren Hypoglykämiegefahr (besonders nachts), der geringeren Variablität und der größeren Flexibilität zu bevorzugen sei.

Das vor knapp zwei Jahren eingeführte Insulin glargin $300 \mathrm{E} / \mathrm{ml}$ führte $\mathrm{zu}$ einer weiteren Verbesserung: Nach einer Metanalyse der EDITION-Studien 1, 2 und 3 zeigte sich, dass die Rate bestätigter $(\leq 70 \mathrm{mg} / \mathrm{dl}$ bzw. $\leq 3,9 \mathrm{mmol} / \mathrm{l})$ und schwerer Hypoglykämien unter Insulin glargin $300 \mathrm{E} / \mathrm{ml}$ bei allen erreichten $\mathrm{HbA}_{1 \mathrm{c}}$-Werten sowohl tagsüber als auch nachts niedriger war als unter Insulin glargin $100 \mathrm{E} / \mathrm{ml}$. Dies war auch bei Patienten mit einer Nierenfunktionsstörung der Fall.

Red.

- Fachpressekonferenz: „Diabetes-Management aus Deutschland", Frankfurt, Februar 2017 (Veranstalter Sanofi)

\section{Otitis externa: nur noch 4-tägige Therapie notwendig}

Eine neue Studie belegt die signifikant überlegene Wirksamkeit von InfectoCiproCort ${ }^{\circledR}$ Ohrentropfen (Ciprofloxacin 0,3\% + Fluocinolonacetonid 0,025\%) im Vergleich zur Monotherapie mit Ciprofloxacin 0,3\% bei Patienten mit akuter Otitis media und Paukenröhrchen [Spektor $Z$ et al. JAMA Otolaryngol Head Neck Surg. 2016]. Durch die Therapie konnte die Dauer bis zum Sistieren der Otorrhö von sieben Tagen unter Ciprofloxacin-Monotherapie auf vier Tage verkürzt werden. Gleichzeitig führte die Kombinationstherapie bei deutlich mehr Patienten zu einer nachhaltigen mikrobiologischen Heilung. Die Studie bestätigt zudem den sicheren Einsatz der Ohrentropfen bei Trommelfelldefekt, $\}$ und dies bereits ab dem 6. Lebensmonat. Das Präparat ist bei Entzündungen des äußeren Gehörgangs voll erstattungsfähig.

Red 